\title{
Hábitos alimenticios de Leporinus friderici (Anostomidae: Teleostei) durante un ciclo hidrobiológico en el río Vaupés, Colombia
}

\author{
Yohana M. Velasco-Santamaría ${ }^{1 *}$, Alexander Torres-Tabares ${ }^{1}$, José A. Ramírez-Saray ${ }^{1}$,
} Pablo E. Cruz-Casallas ${ }^{2}$, Juan A. Ramírez-Merlano ${ }^{2}$, Érica Quiroga-Sanchez ${ }^{3}$

\& Elizabeth Aya-Baquero ${ }^{4}$

1. Universidad de los Llanos, Grupo de Investigación en Biotecnología y Toxicología Acuática y Ambiental (BioTox), km 12 vía Puerto López, Villavicencio - Meta, Colombia; ymvelascos@unillanos.edu.co,

alexander.torres@unillanos.edu.co,saraymvz@gmail.com

2. Universidad de los Llanos, Grupo de Investigación sobre Reproducción y Toxicología de Organismos Acuáticos (GRITOX), km 12 vía Puerto López, Villavicencio - Meta, Colombia; pecruzcasallas@unillanos.edu.co,

jramirezmerlano@unillanos.edu.co

3. Gobernación del Vaupés, Secretaría de Agricultura, Medio Ambiente, Vivienda, Minería y Desarrollo Turístico, Calle 15 \#14 - 18 Mitú - Vaupés, Colombia; erica.quiroga@gmail.com

4. Universidad de los Llanos, Facultad de Ciencias Básicas e Ingeniería, Grupo Biorinoquía, km 12 vía Puerto López, Villavicencio - Meta, Colombia; elizabeth.aya@unillanos.edu.co

Recibido 25-I-2016. Corregido 25-I-2017. Aceptado 24-II-2017.

\begin{abstract}
Feeding habits of Leporinus friderici (Anostomidae: Teleostei) during a hydrobiological cycle in Vaupés River, Colombia. The most important fish species of the Vaupes river is known as a Warakú tres puntos (Leporinus friderici), the most representative migratory Characidae from the Vaupés state, Colombia, with a significant importance in food security of indigenous communities. To contribute with the knowledge of its basic biology, we determined the habits and food preferences of Leporinus friderici, during one hydrobiological cycle (March 2014 to May 2015) in the Vaupés river, Colombia. Three sites were sampled, two of them along the Vaupés river (Yacayacá and Santa Cruz communities) and the other one in the Cuduyarí river, tributary of the Vaupés river (Piracemo community). Physical and chemical water parameters from the surface and depth level of the river were determined. Stomach contents from 316 fish were analyzed by frequency of occurrence (FO), gravimetric index, vacuity index (VI) and relative importance index (RII). Vaupés's average depth in ascending water period was of $3.7 \pm 0.6 \mathrm{~m}$, during rainy season of $5.9 \pm 1.4 \mathrm{~m}$, in descending water period of $4.6 \pm 1.3 \mathrm{~m}$ and during dry season of $2.4 \pm 1.0 \mathrm{~m}$. The higher acidity and turbidity were observed during the ascending water period. The average VI was $14.5 \%$ for the period, but it reached $40 \%$ in descending waters. In general, a FO of plant material was the most frequent (44.4-66.7 \%), followed by insects (21.1-33.3\%, secondary diets). The gravimetric indices showed that plant material was the most consumed item, followed by insects, and in almost in equal proportion, invertebrates and animal material. The differences in diet composition confirm the opportunistic nature of this species with a predominance of omnivorous habits. This study can be used as an integral part of the feeding ecology of $L$. friderici knowledge, in order to develop strategies for its protection in the Vaupés region because of its importance for local communities. Rev. Biol. Trop. 65 (2): 827-842. Epub 2017 June 01.
\end{abstract}

Key words: amazon, stomach contents, indigenous communities, habitat, fish, Warakú.

El departamento de Vaupés es una parte de la región de la amazonia Colombiana y posee una extensión que representa el $11.4 \%$ de los $476000 \mathrm{~km}^{2}$ de la región Amazónica. Cuenta con una riqueza cultural representada en sus más de 65 comunidades indígenas y la biodiversidad reflejada en una gran cantidad de especies de plantas, mamíferos, reptiles y peces.

Dentro de las familias ícticas representativas del departamento, se encuentra 
la Anostomidae con 87 especies registradas en Centro y Suramérica (Garavello \& Britski, 2003). El género Leporinus pertenece a esta familia y es parte fundamental de la dieta de las comunidades indígenas de la región. Peces como el L. friderici (Bloch, 1794), llamado comúnmente en la región como Warakú tres puntos, es una de las especies con mayor incidencia y aceptabilidad por parte de estos pobladores, convirtiéndose en una de las principales fuentes de proteína animal y parte esencial para su seguridad alimentaria. Son especies reofílicas (migran a lo largo de los ríos para su reproducción) que predominan en ambientes lóticos y que pueden reproducirse en ambientes lénticos o semilénticos de planicies inundables (Agostinho, Hahn, Gomes, \& Bini, 1997; Vazzoler, Suzuki, Marques, \& PérezLizama, 1997).

En la Orinoquia y Amazonia se encuentra preferiblemente en ríos y caños de aguas claras y negras, también en planicies de inundación. Específicamente en el Amazonas, esta especie se encuentra en arroyos y ocupan las partes más profundas y oscuras, nadan activamente en contracorriente desplazándose a largas distancias. En el Orinoco, se encuentra en lagunas y caños pequeños durante época de verano; en época de invierno en bosques inundables y zonas de rebalse (Novoa, 2002; Lasso, 2004).

Los informes estadísticos de la Corporación Colombia Internacional (CCI, 2009) reportan que para el año 2008 en el departamento de Vaupés se desembarcaron 18 toneladas de pescado, de 32 especies, la mayoría de ellas de pequeño tamaño, pertenecientes principalmente a las familias Cichlidae, Characidae y Pimelodidae. De la captura total, la especie más importante pertenece al grupo de Leporinidos conocidos como Warakú (Leporinus sp.), con una representación del $20 \%$ del total de peces comercializados para el año 2008, evidenciando una significativa disminución de la población en los cuerpos de agua natural de la región del Vaupés debido a su sobreexplotación. El Leporinus friderici presenta cuerpo fusiforme, boca terminal con cuatro dientes truncados, de 37 a 39 escamas en la línea lateral, su coloración es oscura en el dorso y clara en el vientre, con tres manchas negras ubicadas en la línea lateral, las aletas dorsal, caudal son hialinas, las dorsales y ventrales de color amarillo (Lasso, 2004).

Debido a la importancia biológica de esta especie para la región del Vaupés es necesario determinar aspectos básicos sobre su ecología trófica. El conocimiento de los hábitos alimenticios en peces, y la descripción y cuantificación de la dieta permiten determinar el nivel trófico en que se encuentran las especies y sus relaciones ecológicas con los demás organismos del ecosistema (Zamudio, Urbano, Maldonado, Bogotá, \& Cortés, 2008). De igual modo, permiten comprender los ciclos energéticos, cómo se utilizan los recursos disponibles en su medio y cuál es la posición que ocupan dentro de la red trófica (Lagler, Bardach, Miller, \& Passino, 1977; Hyslop, 1980; Krebs, 1989; Torres-Rojas, 2011; Ramírez-Herrejón, Castañeda-Sam, Moncayo-Estrada, CaraveoPatiño, \& Balart, 2013).

Los hábitos alimenticios de una especie son de gran importancia para conocer su biología básica y características importantes como la dentición, el número de ciegos pilóricos, la forma y longitud del tracto digestivo (Barón, 2006). Al considerar la relevancia de dichas características, el objetivo del presente estudio consistió en describir los hábitos alimenticios y las interacciones tróficas existentes con el hábitat de Leporinus friderici, capturados durante un ciclo hidrobiológico en el río Vaupés, Colombia.

\section{MATERIALES Y MÉTODOS}

Área de estudio: Los muestreos fueron realizados en tres puntos equidistantes a lo largo del río Vaupés (Colombia) y sus afluentes, estos se llevaron a cabo mensualmente durante 24 horas por cada punto de muestreo, durante un ciclo hidrobiológico comprendido desde marzo de 2014 hasta mayo de 2015. Los puntos geográficos de muestreo fueron determinados considerando la abundancia de peces del género Leporinus sp., la accesibilidad a las zonas de muestreo y la distancia 
entre cada punto. Los lugares de muestreo fueron: a) Comunidad indígena de Yacayacá, localizada al sur-occidente $\left(01^{\circ} 05^{\prime} 00,5^{\prime \prime} \mathrm{N}\right.$ - $070^{\circ} 28^{\prime} 19,4$ "' W) a $12.5 \mathrm{~km}$ de la cabecera municipal de Mitú en la parte alta del río Vaupés; b) Piracemo localizado al nor-occidente $\left(01^{\circ} 19^{\prime} 55,8^{\prime \prime} \mathrm{N}-70^{\circ} 23^{\prime} 00,8^{\prime \prime} \mathrm{W}\right)$ a $6.1 \mathrm{~km}$ de la ciudad de Mitú ascendiendo por el caño Cuduyarí hasta las inmediaciones de la comunidad indígena de Piracemo y, c) el tercer punto se encuentra al sur-oriente a $28.3 \mathrm{~km}$ de la ciudad de Mitú $\left(01^{\circ} 09^{\prime} 27^{\prime \prime} \mathrm{N}-70^{\circ} 00^{\prime} 24,3^{\prime \prime} \mathrm{W}\right)$ en la parte baja del río Vaupés, en inmediaciones de las comunidades indígenas de Santa Cruz,
Tayazú, Puerto Paloma y Puerto Esperanza, las cuales son algunas de las comunidades indígenas que conforman la zonal indígena de AATICAM (Asociación de Autoridades Tradicionales Indígenas Aledañas a Mitú). (Fig. 1).

Descripción del hábitat de captura: con el fin de determinar las características del hábitat de la especie en el río Vaupés y sus afluentes, en cada uno de los puntos de muestreo (Yacayacá, Piracemo y Santa Cruz), se procedió a tomar muestras de agua superficial y profunda (fondo, esta última variaba con cada punto de muestreo), por medio de una botella

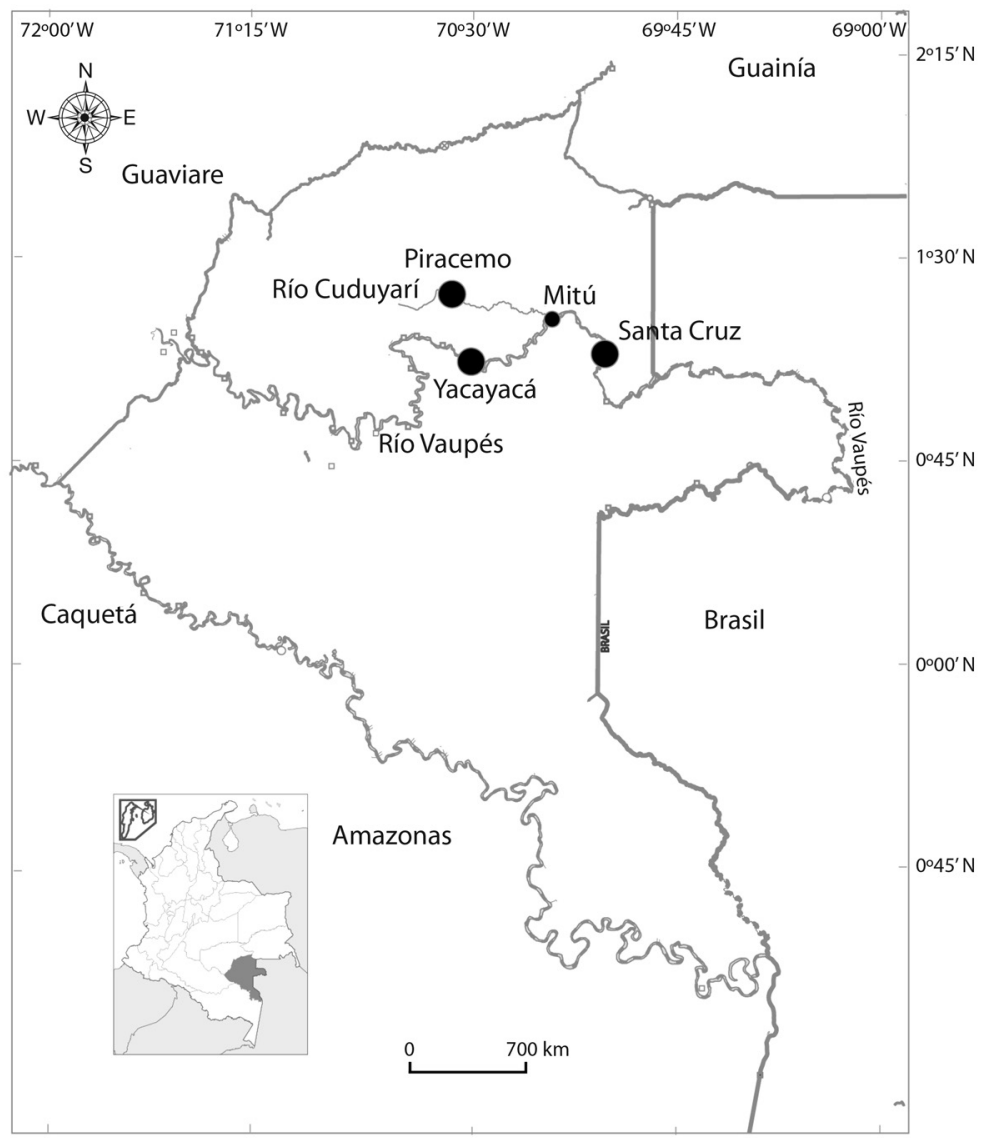

Fig. 1. Localización de los puntos de muestreo, cuerpos de agua aledaños a las comunidades indígenas de Yacayacá $\left(01^{\circ} 05^{\prime} 00,5^{\prime \prime} \mathrm{N}-070^{\circ} 28^{\prime} 19,4{ }^{\prime} \mathrm{W}\right)$, Piracemo $\left(01^{\circ} 19^{\prime} 55,8^{\prime \prime} \mathrm{N}-70^{\circ} 23^{\prime} 00,8^{\prime \prime} \mathrm{W}\right)$ y Santa Cruz $\left(01^{\circ} 09^{\prime} 27^{\prime} \mathrm{N}-70^{\circ} 00^{\prime} 24,3^{\prime \prime} \mathrm{W}\right)$ en el departamento de Vaupés, Colombia.

Fig. 1. Location of sampling points, water bodies close by the indigenous communities of Yacayacá $\left(01^{\circ} 05^{\prime} 00,5^{\prime \prime} \mathrm{N}\right.$ $070^{\circ} 28^{\prime} 19,4$ " W), Piracemo $\left(01^{\circ} 19^{\prime} 55,8^{\prime \prime} \mathrm{N}-70^{\circ} 23^{\prime} 00,8^{\prime \prime W}\right)$ y Santa Cruz $\left(01^{\circ} 09^{\prime} 27^{\prime \prime} \mathrm{N}-70^{\circ} 00 ` 24,3^{\prime \prime} \mathrm{W}\right)$ at the department of Vaupés, Colombia. 
de Van Dorn de 3 L, para medir parámetros como temperatura y $\mathrm{pH}$ (Hanna Instruments HI-9214, UK), transparencia (disco de Secchi; Tyler, 1968), amonio y oxígeno disuelto (Hach Nine-Parameter Test Kit, Model FF-1A Iowa, USA). Así mismo, en cada lugar de captura fue registrado de acuerdo al tipo de cuerpo de agua (cauce principal, laguna, rebalse, laguna, raudal, entre otros), profundidad, naturaleza del fondo (rocoso, lodoso, arenoso, hojarasca, entre otros) y vegetación ribereña.

Material biológico: se analizaron 316 contenidos estomacales de la especie Leporinus friderici, cuya clasificación taxonómica fue confirmada en el Laboratorio de Biología de la Universidad de los Llanos. Se realizaron aleatoriamente muestreos cada mes con la finalidad de incluir la variabilidad natural en las preferencias alimentarias de la especie, considerando cambios estacionales. Para este caso, fueron consideradas las variaciones durante el ciclo hidrobiológico así como el lugar de muestreo (Hajisamaea, Choua, \& Ibrahimb, 2003; Kellnreitner, Pockberger, \& Asmusl, 2012). La captura de los peces fue realizada por pescadores nativos de las comunidades indígenas aledañas a cada lugar de muestreo, usando en todos los meses y zonas de muestreo artes de pesca pasivos como el chinchorro, anzuelo, zagaya (elemento en forma de arpón), cacurí (trampa para peces en forma de canasto) y matapi (encierro realizado para que los peces en época de aguas altas queden atrapados) con faenas de pesca realizadas entre las cinco (05) y diez (10) am (Sostoa, Garcia De Jalón, \& Garcia-Berthou, 2005). Las capturas reflejaron el siguiente número de ejemplares capturados durante el año 2014 marzo $(\mathrm{n}=21)$, abril $(\mathrm{n}=$ $9)$, mayo $(n=18)$, junio $(n=13)$, julio $(n=15)$, agosto $(\mathrm{n}=12)$, septiembre $(\mathrm{n}=38)$, octubre $(\mathrm{n}$ $=36)$, noviembre $(\mathrm{n}=25)$ y diciembre $(\mathrm{n}=23)$, y durante el año 2015 el número de peces fue: enero $(n=9)$, febrero $(n=20)$, marzo $(n=21)$, abril $(\mathrm{n}=34)$ y mayo $(\mathrm{n}=22)$.
Procesamiento de muestras y análisis de contenido estomacal: una vez capturados los peces fueron sumergidos en una solución de tranquilizante 2-fenoxietanol $(0.5 \mathrm{~mL} / \mathrm{L})$, e insensibilizados por corte medular. De cada ejemplar se registró la longitud total (LT) y estándar (LE) expresada en $\mathrm{cm}$ por medio de un ictiómetro, así como el peso corporal en gramos (g) con una balanza de precisión $0.1 \mathrm{~g}$ (Ohaus Traveler TA501 $\pm 0.1 \mathrm{~g}$ ); posteriormente, fueron disectados y extraído el estómago, el cual fue preservado en formol al $10 \%$ en frascos plásticos rotulados.

En el Laboratorio de Biología de la Universidad de los Llanos, se registró el peso del estómago lleno y vacío con una balanza analítica de precisión (Ohaus Explorer Pro EP214C- $0.0001 \mathrm{~g}$ ). Los organismos visualizados en los estómagos se determinaron hasta nivel de orden, con el empleo de claves especializadas para cada grupo de alimento (Heinz \& Dieter, 1987; Needham \& Needham, 1982; Roldán \& Ramírez, 2008). Los datos obtenidos fueron analizados por los métodos cualitativos y cuantitativos.

De acuerdo con la técnica de Longart, Acosta, Parra y Lista (2011), el contenido estomacal se extendió en una caja de Petri, para luego examinarlo bajo el estereomicroscopio, donde los alimentos se separaron, identificaron y agruparon en seis categorías, así:

1. Material vegetal (hojas, flores, frutos, semillas, corteza, madera).

2. Insectos como Blattodea, Coleoptera, Diptera, Ephemeroptera, Anisopteros, Lepidoptera, Ortoptera, Hemiptera e Hymenoptera.

3. Restos de invertebrados como antenas, caparazones y demás estructuras corporales de los grupos de: Nematoda, Pulmonata, Annelida, Chilopoda, Decapoda, Trichoptera y Araneae.

4. Restos de vertebrados: restos de peces (trozos de pescado, espinas, escamas). 
5. Material particulado: material mineral como limo, arena detritos.

6. Material digerido no identificado (MDNI).

Método cualitativo: Frecuencia de ocurrencia (FO). $\mathrm{FO}=\mathrm{n} / \mathrm{N} * 100$; se estimó con la ecuación donde $\mathrm{n}$ es el número de estómagos que contienen una presa determinada y $\mathrm{N}$ el número total de estómagos con alimento. Con este método se distinguieron tres categorías de presas: accidentales $\mathrm{FO}<10$, secundarias $10<$ FO $<50$ y preferenciales FO $>50$ (Franco \& Kmbashirullah, 1992).

Se presentan a continuación los resultados de los métodos cuantitativos.

Índice gravimétrico $(\mathbf{W})$ : se estimó empleando la siguiente fórmula (Hyslop, 1980)

$\mathrm{W}=$ Peso total de un ítem específico A presente en un estómago * 100 Peso total del contenido estomacal (g)

Coeficiente de vacuidad (CV): se estimó empleando la fórmula de Zamudio et al. (2008).

$\mathrm{CV}=\underline{\text { Número de estómagos vacíos } * 100}$

Número de estómagos examinados

Índice de Importancia Relativa (IIR): se estimó empleando la fórmula propuesta por Yáñez-Arancibia et al. (1985).

$\mathrm{IIR}=\% \mathrm{FO} * \% \mathrm{Gv} / 100$

$\mathrm{FO}=\%$ de frecuencia de ocurrencia de un tipo de alimento, en el total de estómagos examinados

$\mathrm{Gv}=\%$ en peso de un tipo de alimento, en el peso total de estómagos examinados.

El rango de 0 a $10 \%$ representa grupos tróficos de importancia relativa baja, de 10 a 40 $\%$ grupos de importancia relativa secundaria y 40 a $100 \%$ grupos de importancia relativa alta.

Con el fin de analizar las fases hidrológicas, los muestreos mensuales fueron agrupados en: aguas ascendentes (marzo y abril de 20142015), altas (mayo a julio de 2014 y mayo de 2015), descendentes (agosto a octubre de 2014) y bajas (noviembre a diciembre de 2014 y enero a febrero de 2015), utilizando como criterio de agrupación la profundidad media del río.
Los datos fueron sometidos a verificación de normalidad y homogeneidad de varianza y posteriormente a un análisis de varianza, utilizando un modelo estadístico completamente aleatorizado y la prueba de $\mathrm{F}$ fue aplicada para verificar la significancia de los cuadrados medios de las fuentes de variación, tomando el nivel de significación $\mathrm{P}<0.05$. Cuando el ANAVAR detectó diferencias significativas entre los diferentes parámetros de evaluación, los promedios fueron separados de acuerdo con el resultado de la prueba de Tukey (Steel \& Torrie, 1988), al mismo nivel de significancia utilizado en el análisis de la varianza. El análisis estadístico se realizó utilizando programa estadístico SAS (versión 9.2, 2009) y GraphPad (versión 5.03, 2009). Así mismo, se calculó el índice de similitud trófica a través del análisis de Bray-Curtis con base al contenido estomacal de los peces muestreados en los tres sitios y las cuatro épocas del ciclo hidrológico, previo transformación de los datos a raíz cuadrada. La matriz de similitud fue sometida a un análisis de Cluster jerárquico. Posteriormente se realizó un análisis de escalado multidimensional (MDS en inglés). Para estos análisis se usó el software PRIMER v6.

\section{RESULTADOS}

Descripción del hábitat de captura y evaluación de parámetros físico-químicos: según el monitoreo mensual realizado a los niveles del río Vaupés se pudo determinar que para los meses de marzo y abril las aguas son ascendentes, de mayo a julio las aguas son altas, de agosto a octubre las aguas son descendentes y de noviembre a febrero son aguas bajas.

Otros parámetros físicos y químicos fueron determinados en los tres sitios de muestreo durante marzo de 2014 a mayo de 2015 (Cuadro 1). La mayor turbidez fue observada en Piracemo en la época de aguas ascendentes siendo estadísticamente diferentes cuando fue comparado con Yacayacá y Santa Cruz en la misma fase hidrológica (Tukey, $\mathrm{P}<0.05$ ). La menor turbidez fue observada en Yacayacá y Santa Cruz en aguas ascendentes y altas. 


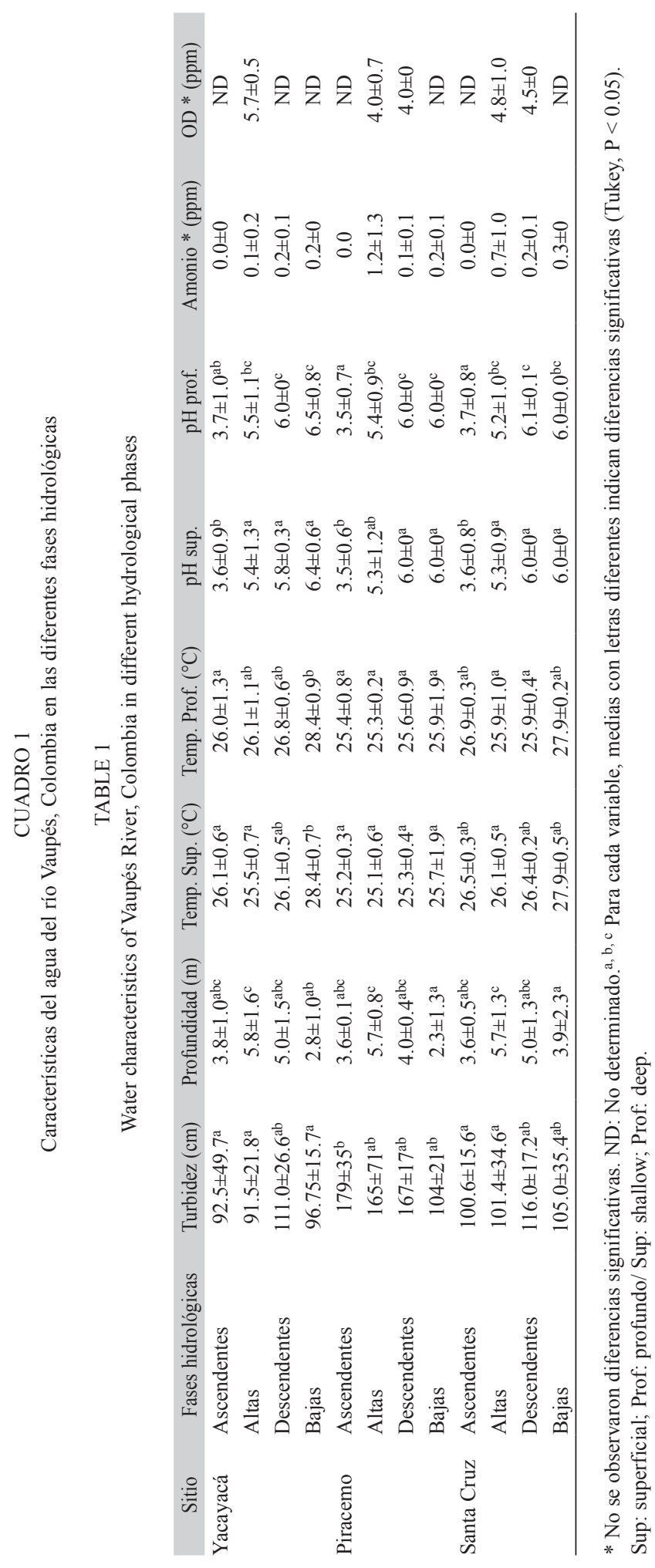


No se observaron diferencias entre los tres puntos de muestreo con respecto a la profundidad del río, el promedio general en época de aguas ascendentes fue de $3.7 \pm 0.6 \mathrm{~m}$, aguas altas de $5.9 \pm 1.4 \mathrm{~m}$, aguas descendentes $4.6 \pm 1.3 \mathrm{~m}$ y en aguas bajas $2.4 \pm 1.0 \mathrm{~m}$. La temperatura superficial y profunda del agua se mantuvo relativamente estable a lo largo de las fases hidrológicas; en general, se observó mayores temperaturas en la época de aguas bajas siendo mayor en Yacayacá $\left(28.4 \pm 0.7{ }^{\circ} \mathrm{C}\right)$. Se observó una mayor acidez del agua en aguas ascendentes $(3.5 \pm 0.8)$ cuando comparado con las demás fases hidrológicas (Tukey, $\mathrm{P}<0.05$ ). Con respecto a la dureza, esta se mantuvo homogénea en todos los sitios de muestreo y durante las fases hidrológicas (17.1 ppm).
Se presenta a continuación los resultados de los análisis de contenido estomacal.

Frecuencia de ocurrencia (FO): cada uno de los ítems analizados se ilustra en la figura 2. En general, se observó una mayor FO de material vegetal en los individuos muestreados (44.4\% consolidado total) clasificando esta dieta como secundaria; no obstante, en Yacayacá aguas bajas el FO fue de $51 \%$, siendo este alimento clasificado como preferencial (Fig. 2B). Por otra parte, los insectos ocuparon el segundo puesto $(21.1 \%$ consolidado total), no obstante, los patrones variaron de acuerdo con el sitio de muestreo, siendo esta dieta categorizada como secundaria. En el punto de Yacayacá, se observó mayor FO en aguas
A Consolidado río Vaupés
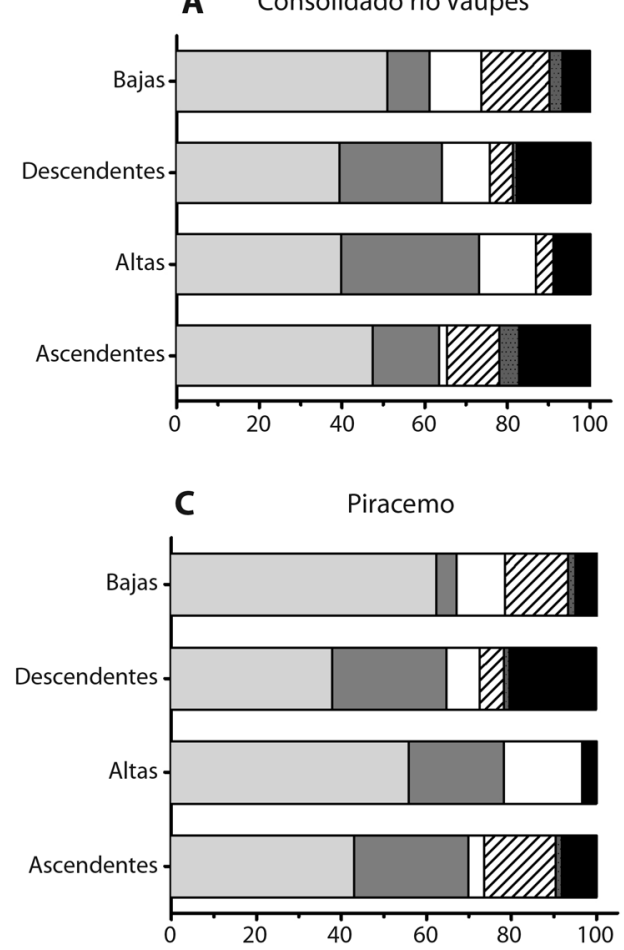

$\square \mathrm{MV}$
B Yacayacá

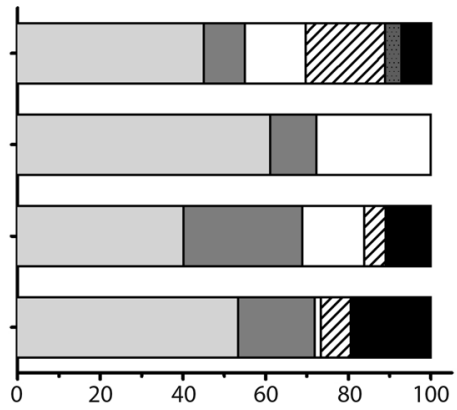

D Santa Cruz

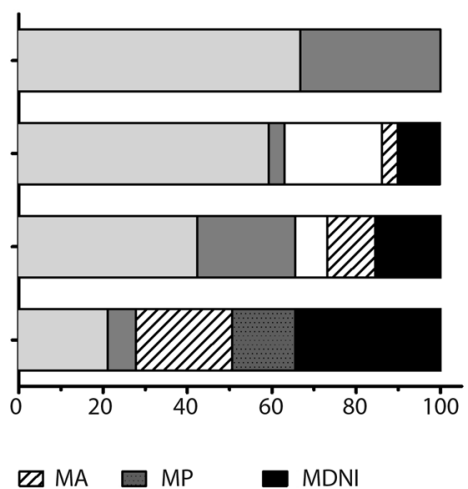

Fig. 2. Frecuencia de ocurrencia (\%) de ítems alimenticios en peces Leporinus friderici muestreado desde marzo 2014 a mayo 2015. A. Valores que consolidan los tres sitios de muestreo. B. Yacayacá. C. Piracemo. D. Santa Cruz.

Fig. 2. Frequency of occurrence (\%) of food items Leporinus friderici fish sampled from the months of March 2014 to May 2015. A. Values that consolidate all three sampling sites. B. Yacayacá. C. Piracemo. D. Santa Cruz. 


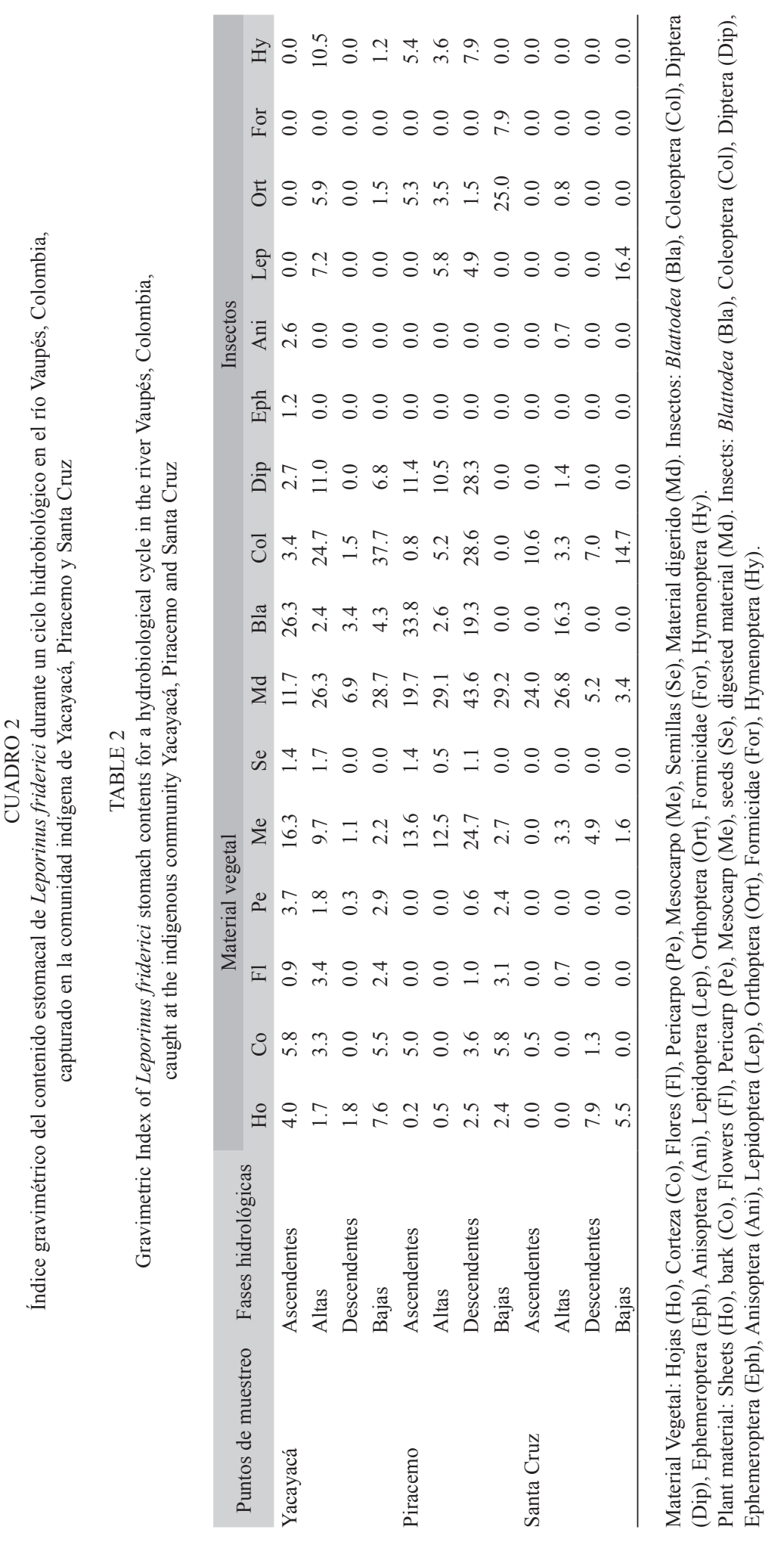


altas $(28.8 \%)$ seguido de aguas ascendentes (18.6 \%, Fig. 2B). En la zona de Santa Cruz, se observaron claras diferencias en la FO de los ítems alimenticios, en época de aguas bajas solo se registró presencia de material vegetal $(66.7 \%)$ e insectos (33.3\%, Fig. 2D). Se observó presencia moderada de material digerido no identificado (MDNI), el cual fue menor en la época de agua bajas (excepto Santa Cruz). En aguas ascendentes y descendentes el MDNI fue categorizado como secundario y en las épocas de aguas altas y bajas tendieron a ser presas accidentales. Las cantidades de ítems de material vegetal, insectos, otros invertebrados, peces, material particulado y MDNI, variaron dependiendo del sitio y la fase hidrológica (Cuadro 2 y Cuadro 3). En general, el material vegetal fue el ítem consumido en mayor cantidad, seguido de los insectos y en casi igual proporción invertebrados y material animal. El material digerido no identificado estuvo presente en un porcentaje significativo.
En los contenidos estomacales de los peces capturados, se observó un consumo importante de mesocarpio, seguido de hojas y corteza. Por su parte, se registró el mayor índice gravimétrico para insectos del género Blattodea, Coleoptera y Diptera (Cuadro 2 y Cuadro 3). Otros invertebrados se determinaron en diferentes proporciones en los contenidos estomacales de los peces capturados, en Yacayacá durante la fase de aguas altas se observó un índice gravimétrico de $13.4,15.7,13.5$ y $14.1 \%$ correspondientes a Nematoda, Pulmonata, Anelida y Trichoptera, respectivamente (Cuadro 2 y Cuadro 3). Por su parte, en Piracemo, los ítems de invertebrados más consumidos fueron nemátodos, anélidos y decápodos, durante la época de aguas descendentes. A diferencia, en la zona de Santa Cruz, los peces consumieron más anélidos y quilópodos en época de aguas altas, y decápodos en aguas descendentes. Los restos óseos de peces se registraron en todos los sitios y fases hidrográficas, pero se observó en mayor

CUADRO 3

Índice gravimétrico del contenido estomacal de Leporinus friderici durante un ciclo hidrobiológico en el río Vaupés, Colombia, capturado en la comunidad indígena de Yacayacá, Piracemo y Santa Cruz

TABLE 3

Gravimetric Index of Leporinus friderici stomach contents for a hydrobiological cycle Vaupés river, Colombia, caught at the indigenous community Yacayacá, Piracemo and Santa Cruz

\begin{tabular}{llcccccccccccc}
\multirow{3}{*}{ Puntos de muestreo } & \multicolumn{1}{c}{ Otros invertebrados } & \multicolumn{1}{c}{ Peces } \\
Yacayacá & Fases hidrológicas & Ne & Pul & Ane & Chi & Dec & Tri & Ara & Pec & Esc & Hue & Mp & Mad \\
& Ascendentes & 0.0 & 0.0 & 0.0 & 0.0 & 0.0 & 0.0 & 0.0 & 0.0 & 0.0 & 21.5 & 0.0 & 41.6 \\
& Altas & 13.4 & 15.7 & 13.5 & 0.0 & 0.0 & 14.1 & 0.0 & 0.0 & 0.0 & 59.4 & 3.0 & 25.0 \\
& Descendentes & 0.0 & 0.0 & 0.0 & 0.0 & 9.9 & 0.0 & 0.0 & 0.0 & 0.0 & 0.0 & 0.0 & 0.0 \\
\multirow{3}{*}{ Piracemo } & Bajas & 0.0 & 0.0 & 0.0 & 0.0 & 0.0 & 0.0 & 0.0 & 0.0 & 0.0 & 57.4 & 61.4 & 53.0 \\
& Ascendentes & 0.0 & 0.0 & 0.0 & 0.0 & 0.0 & 0.0 & 0.0 & 6.7 & 4.2 & 43.0 & 66.7 & 57.2 \\
& Altas & 8.6 & 0.0 & 0.0 & 0.0 & 0.0 & 0.0 & 0.0 & 0.0 & 0.0 & 0.0 & 0.0 & 40.7 \\
& Descendentes & 3.9 & 0.0 & 18.2 & 0.0 & 19.2 & 0.0 & 0.0 & 9.4 & 0.0 & 74.9 & 66.7 & 98.8 \\
\multirow{3}{*}{ Santa Cruz } & Bajas & 0.0 & 0.0 & 0.0 & 0.0 & 0.0 & 0.0 & 0.0 & 0.0 & 0.0 & 0.0 & 0.0 & 0.0 \\
& Ascendentes & 0.0 & 0.0 & 0.0 & 0.0 & 0.0 & 0.0 & 0.0 & 0.0 & 0.0 & 0.0 & 0.0 & 1.8 \\
& Altas & 0.0 & 0.0 & 28.1 & 6.3 & 0.0 & 0.0 & 0.0 & 0.0 & 0.0 & 40.6 & 22.0 & 29.6 \\
& Descendentes & 0.0 & 0.0 & 0.0 & 0.0 & 12.7 & 0.0 & 0.0 & 0.0 & 0.0 & 23.6 & 0.0 & 1.8 \\
& Bajas & 0.0 & 0.0 & 0.0 & 0.0 & 0.0 & 0.0 & 0.0 & 0.0 & 0.0 & 0.0 & 0.0 & 0.0 \\
\hline
\end{tabular}

Otros invertebrados: Nematoda (Ne), Pulmonata (Pul), Annelida (Ane), Chilopoda (Chi), Crustacea (Cru), Decapoda (Dec), Trichoptera (Tri), Araneae (Ara), Peces: Peces digeridos y parcialmente digeridos (Pec), Escamas (Esc), Huesos (Hue), Material particulado (Mp) y Material animal digerido (Mad).

Other invertebrates: Nematoda (Ne), Pulmonata (Pul), Annelida (Ane), Chilopoda (Chi), Crustacea (Cru), Decapoda (Dec), Trichoptera (TRI), Araneae (Ara) Fish: Fish digested and partially digested (Pec), Scales (Esc), Bones (Hue), Particulate matter (PM) and Digested animal material (Mad). 
A Consolidado río Vaupés
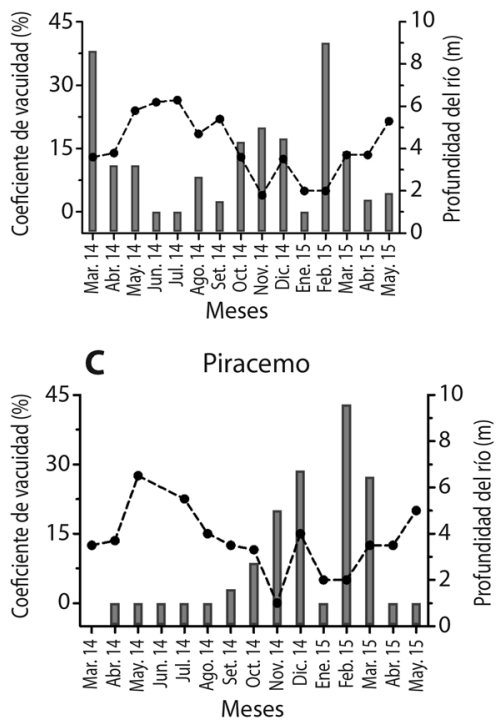

B Yacayacá
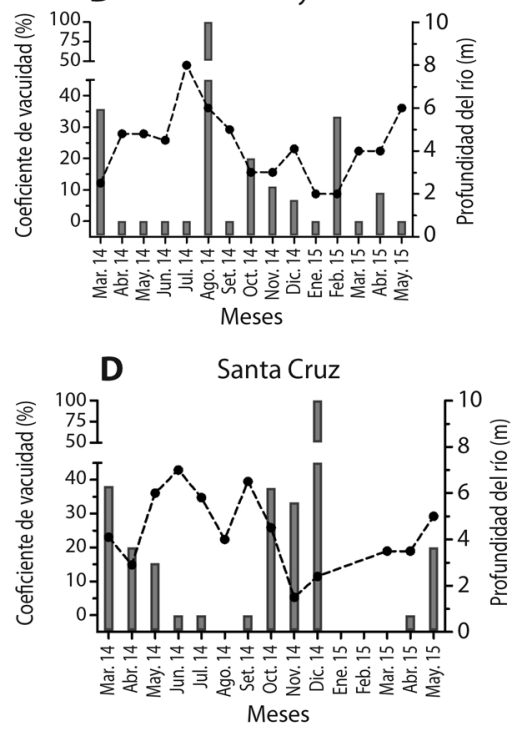

Fig. 3. Coeficiente de vacuidad (CV, barras, eje Y izquierdo) de Leporinus friderici muestreados desde marzo 2014 a mayo 2015 contrastado con los resultados de profundidad del río Vaupés o sus afluentes (m, línea punteada, eje Y derecho). A. Valores consolidando los tres sitios de muestreo. B. CV (\%) y profundidad del río Vaupés en el sitio de Yacayacá. C. CV (\%) y profundidad del río Cuduyarí afluente del río Vaupés en el sitio de Piracemo. D. CV (\%) y profundidad del río Vaupés en la zona de Santa Cruz.

Fig. 3. Vacuity coefficient (CV, bars, Y left axis) of Leporinus friderici sampled from March 2014 to May 2015 contrasted with the results of Vaupés river depth ( $\mathrm{m}$, dotted line, Y right axis) or its tributaries. A. Values consolidating the three sampling sites. B. CV (\%) and depth of the Vaupes river in Yacayacá site. C. CV (\%) and depth at Cuduyarí Vaupes River tributary of the river Piracemo site. D. CV (\%) and depth of the Vaupes river at Santa Cruz site.

proporción en Piracemo en aguas descendentes (74.9 \%) y Yacayacá en aguas altas $(59.4 \%)$.

Coeficientes de vacuidad (CV): durante el ciclo hidrobiológico $(n=316)$ fue del 14.5 $\%$; y se registró el CV más alto (40\%) entre los meses de octubre a diciembre. Los niveles del río muestran a los meses de marzo y abril como aguas ascendentes, los meses de mayo a julio como aguas altas, los meses de agosto a octubre como aguas descendentes y los meses de noviembre a febrero como aguas bajas (Fig. 3).

Índice de importancia relativa (IIR): en la Figura 4A, se observa el consolidado del IIR, evidenciándose para el material vegetal (MV) valores por encima del $40 \%$, indicando su alta importancia relativa dentro de la dieta del Warakú tres puntos (Leporinus friderici). Los insectos se clasifican como grupos tróficos ocasionales o de importancia relativa secundaria con valores del índice entre 10 y $30 \%$ en aguas descendentes y altas; de igual forma, para el ítem material digerido no identificado (MDNI) un índice de $20 \%$ en los meses de aguas descendentes y ascendentes. Los demás ítems como: otros invertebrados, material animal (MA) y material particulado (MP) registran índices de 8 al $10 \%$, clasificados como grupos tróficos circunstanciales y de baja importancia relativa. El grupo trófico material animal (MA) en los meses de aguas bajas aumentó en su índice por encima del $16 \%$ categorizándolo para estos meses como grupo trófico de importancia relativa secundaria.

Análisis de similitud trófica: el análisis de cluster de Bray-Curtis y el escalado multidimensional, permitieron identificar el agrupamiento en dos grupos con un porcentaje 

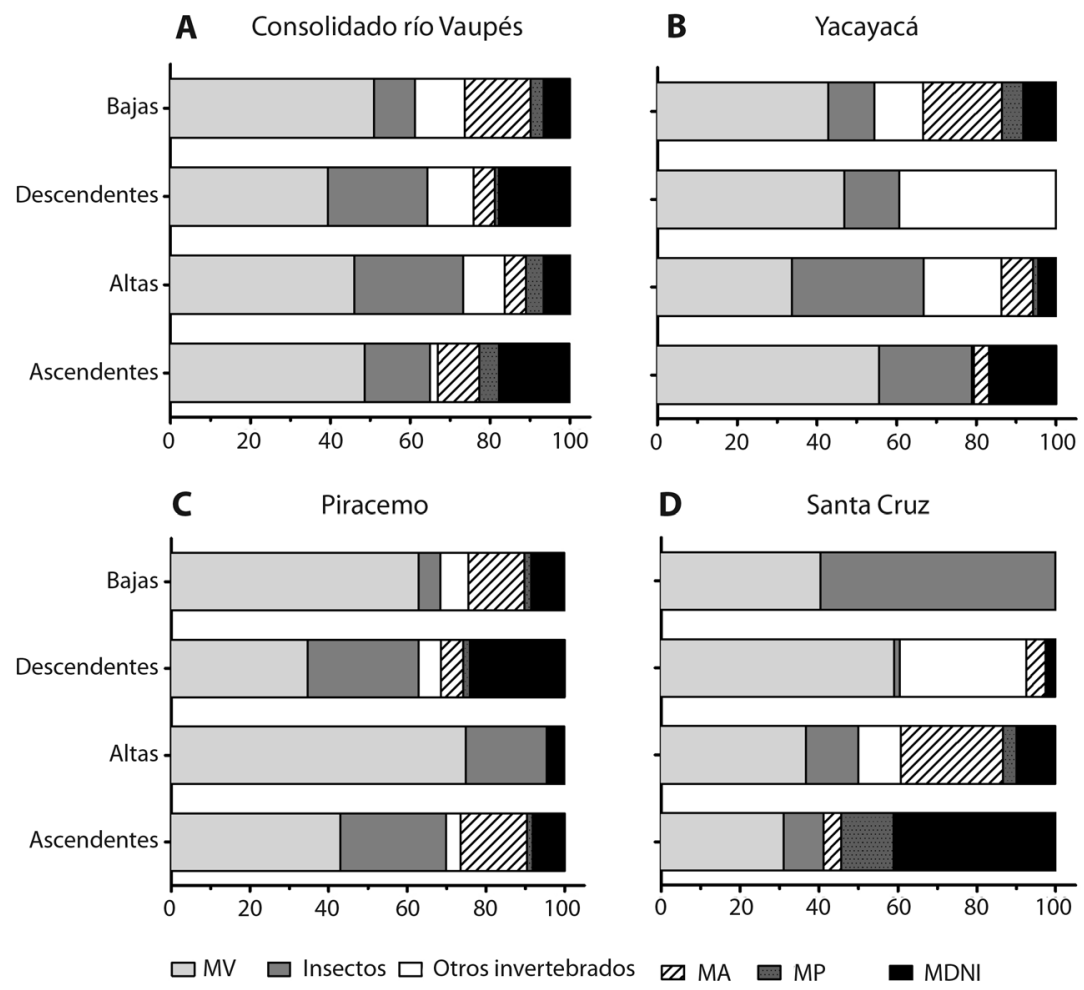

Fig. 4. Índice de Importancia Relativa (IIR) (\%) de ítems alimenticios en peces Leporinus friderici muestreado desde los meses de marzo de 2014 a mayo de 2015. A. Valores consolidando los tres sitios de muestreo. B. Yacayacá. C. Piracemo. D. Santa Cruz.

Fig. 4. Relative importance index (IIR) (\%) of food items Leporinus friderici fish sampled from the months of March 2014 to May 2015. A. Values that consolidate all three sampling sites. B. Yacayacá. C. Piracemo. D. Santa Cruz.

de similitud superior al $60 \%$ : el grupo 1 correspondiente a aguas ascendentes y altas para los tres sitios de muestreo y el grupo 2 a aguas descendentes y bajas para los tres sitios de muestreo, a excepción del punto aguas descendentes en Piracemo, que tuvo un comportamiento similar al grupo 1 (Fig. 5).

\section{DISCUSIÓN}

En el presente estudio se evidenció la presencia de suelos arenosos con presencia de palizadas en el río Vaupés, a excepción de la zona de Santa Cruz, cuyos suelos son pedregosos con poca arena. Las aguas del río Vaupés se tiñen con taninos provenientes de la descomposición del follaje, por los que se les denomina ríos negros (Lasso \& Sánchez-Duarte, 2011).
La mayor acidez del agua observada en aguas ascendentes, probablemente está asociada con la presencia de taninos y material vegetal en descomposición durante esta época.

La FO de MDNI en la mayoría de los sitios puede estar asociada a las artes de pesca usadas por los indígenas. Tradicionalmente, las comunidades indígenas usan como artes de pesca el anzuelo, zagaya, chinchorro (artes de pesca activos), cacuri y matapi (artes de pesca pasivos). A este respecto, las artes de pesca pasivas conllevan a una mayor permanencia del pez en dichos elementos, favoreciendo probablemente una mayor digestión del contenido estomacal, y por tanto la presencia de material digerido.

En reportes realizados del Leporinus friderici, en el río Paraná, se encontró que esta especie consume gran cantidad de insectos terrestres 

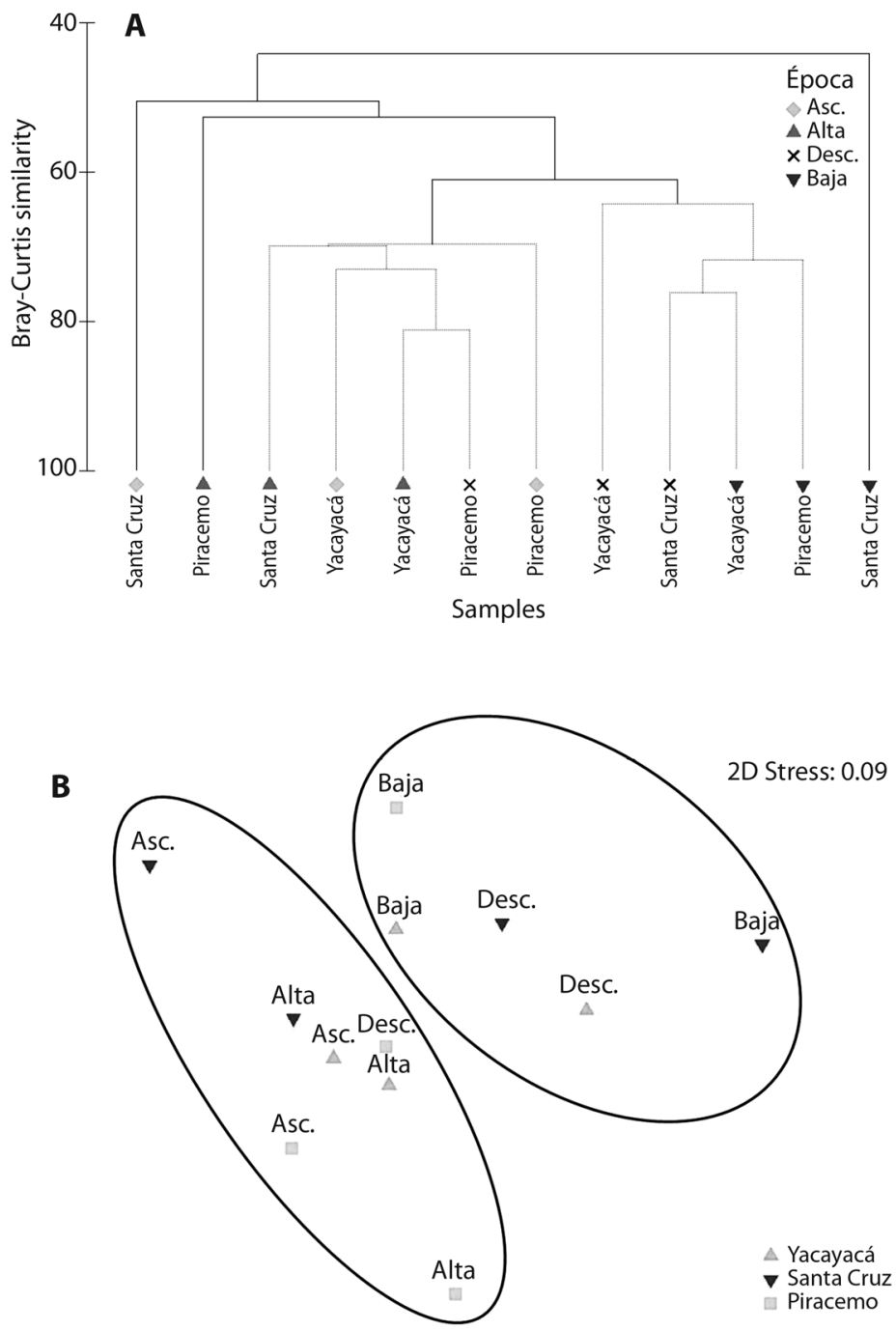

Fig. 5. Análisis de cluster jerárquico de Bray-Curtis y MDS con base en el contenido estomacal de peces Leporinus friderici muestreados desde marzo 2014 a mayo 2015. A. Similitud superior al 60 \%; B. MDS mostrando el agrupamiento dentro de 2 grupos cada uno con similitudes en sus ítems alimenticios. Asc: aguas ascendentes, Alta: aguas altas, Desc: aguas descendentes, Baja: Aguas bajas.

Fig. 5. Hierarchical cluster analysis (Bray-Curtis) and MDS based on stomach content of Leporinus friderici sampled during the months of March 2014 to May 2015. A. Similarity higher than $60 \%$; B. MDS showing grouping between two groups each one with similarities in the feeding items. Asc: ascending water level, High: high water level, Desc: descending water levels, Low: low water levels.

(termitas, Isoptera y hormigas, Hymenoptera) durante la época de aguas ascendentes (Hahn, Andrian, Fugi, \& De Almeida, 1997), como lo demuestra este estudio. La presencia de grandes cantidades de estos insectos en la dieta de la especie en este período, se explica por la inundación de los termiteros y hormigueros durante el aumento de los niveles de las aguas del río Vaupés. Sin embargo, se encontró que su importancia en la dieta se redujo en $L$. friderici cuando se agotó la disponibilidad de alimento, siendo reemplazado con peces nativos, algas filamentosas, malezas acuáticas, frutos y semillas, entre otros durante la época de aguas altas 
(Santos, 1982) evidenciándose este comportamiento en el presente trabajo correspondiente a los peces capturados en la comunidad de Santa Cruz. Las diferencias en la composición de la dieta entre los sitios de captura de las comunidades de Yacayacá, Piracemo y Santa Cruz, confirman la naturaleza oportunista de esta especie, que debido a la variedad de ítems o grupos tróficos ingeridos, pueden ser clasificados como especie omnívora con preferencia por vegetales (Agostinho et al., 1997).

La mayor oferta de recursos alimenticios ocurrió durante el período de nivel de aguas altas del río Vaupés, teniendo en cuenta que el incremento del caudal y el escurrimiento de las aguas de lluvia, arrastran sedimentos y materiales biológicos, aumentan la oferta de recursos alóctonos a los ríos (Mendonça, Magnusson, \& Zuanon, 2005), permitiendo a su vez mayor disponibilidad alimenticia para los peces. En este estudio se determinó para el mes de marzo un $\mathrm{CV}$ de $100 \%$, mientras que durante el mes de noviembre se registró el menor CV (13.0\%). Por su parte, Pérez-Mayorga \& Prada-Pedreros (2011) reportaron en el período hidrológico de nivel de aguas bajas un coeficiente de vacuidad de $7.1 \%$, mientras que en el período hidrológico de nivel de aguas altas un CV de $1.5 \%$. Sin embargo, Villadiego, Ortiz-Villafañe y AtencioGarcía (2004) reportaron un índice de vacuidad $>42.0 \%$ durante todo el año.

Para los meses de agosto y diciembre, en la comunidad de Yacayacá y Santa Cruz, respectivamente, se observó un coeficiente de vacuidad de $100 \%$, para los ejemplares capturados (dos ejemplares en total con el estómago vacío). La poca cantidad de animales capturados está relacionada probablemente con el patrón de migración de estos individuos, el cual se ha encontrado que modifica sus hábitos alimenticios y su capacidad de reproducirse (Maldonado-Ocampo et al., 2005). Esta especie tiene una corta migración debido a los numerosos raudales del río Vaupés, los cuales solo permiten su migración desde el río Vaupés hasta el río Unilla e Itilla en el departamento del Guaviare (Lasso, 2004). Adicionalmente, estos altos índices de vacuidad podrían estar asociados a los métodos de captura, muestreos en horas de ayuno o el tiempo transcurrido entre el momento de pesca y su muestreo.

De acuerdo al índice de importancia relativa (IIR) para este estudio, se encontró el material vegetal (MV) como grupo trófico de gran importancia dentro de la dieta del Warakú tres puntos, sin embargo, en algunas comunidades como Yacayacá, se pudo encontrar otros grupos tróficos de importancia ocasional como los insectos, material digerido no identificado (MDNI), restos de invertebrados y vertebrados. Debido a que no se encontraban en igual porcentaje de importancia para todos los meses durante el ciclo hidrobiológico, se observa que para cada mes, el material vegetal compartió diferente grupo trófico de gran importancia en la dieta de esta especie, ratificando que la especie objeto de estudio es oportunista, de dieta ocasional omnívora. El género Leporinus presenta una amplia gama de grupos tróficos, por lo cual se les puede considerar omnívoros (Castagnolli, 1992). De igual forma, Casas, Lozano-Largacha y Rivas (2007) reportaron al Leporinus muyscorum como especie omnívora con tendencias a herbívora, prefiriendo dentro de sus dietas grupos tróficos como material vegetal (hojas, frutos, granos y semillas), insectos, restos de peces y detritos. En otras especies como el Leporinus elongatus, especie omnívora, sus hábitos alimenticios están determinados por la disponibilidad de alimentos en el medio ambiente, encontrándose dentro de su dieta en aguas ascendentes hojas, frutas y semillas e insectos como termitas, así como microcrustáceos (Durães, Pompeu, \& Godinho, 2001).

El patrón observado con los insectos puede deberse a que en época ascendente y aguas altas, los nichos de los insectos son arrastrados por la corriente y la posibilidad de ser consumidos y encontrarse en los estómagos es mayor. La zona de Santa Cruz es altamente caudalosa con presencia de varios raudales lo cual podría explicar la razón por la cual solo se observó material vegetal e insectos en los contenidos estomacales, ya que los invertebrados pueden tener dificultad de adherirse o mantener sus hábitats en condiciones con alta corriente. 
En esta región el comportamiento del caudal del río Vaupés se encuentra directamente relacionado con los niveles de escorrentía, los cuales dependen de la época del año; durante la época de aguas ascendentes se reporta un caudal medio en Mitú de $1294.2 \mathrm{~m} 3 / \mathrm{seg}$, y en área de raudales como Santa Cruz puede ser mayor a $2700 \mathrm{~m}^{3} / \mathrm{seg}$ (PGAR, 2011).

El análisis de similitud trófica permitió identificar que los contenidos estomacales de L. friderici tienden a ser similares de acuerdo a la época de aguas, donde las épocas de aguas bajas y descendentes se agruparon de acuerdo a los ítems, y la época de aguas ascendentes y altas tuvieron un comportamiento de agrupamiento similar.

Las diferencias en los parámetros evaluados de los diferentes puntos de muestreo se deben específicamente a sus condiciones geográficas, que influyen directamente sobre la disponibilidad de alimento, patrones de migración e incluso sobre el comportamiento reproductivo de los peces. La zona hídrica de Yacayacá se encuentra ubicada al sur occidente de la cabecera municipal de Mitú y se caracteriza por tener abundante materia orgánica en descomposición fruto de los árboles y hojas caídos en el río. La zona hídrica de Piracemo al nor-occidente del departamento, está ubicada en la cuenca del caño Cuduyari, afluente del río Vaupés y se caracteriza por sus aguas tranquilas y gran diversidad de organismos acuáticos y la zona hídrica de Santa Cruz, ubicada al sur oriente del departamento, se caracteriza por sus grandes y caudalosos raudales los cuales afectan la migración natural de los peces y sus patrones alimenticios.

Este estudio puede ser utilizado como parte integral del conocimiento de la ecología trófica de la especie, información que será útil para la creación de estrategias para la protección del Leporinus friderici, el cual ha disminuido en número de manera significativa de los cuerpos de agua natural de la región del Vaupés debido a su sobreexplotación. El presente estudio evidenció que Leporinus friderici es una especie oportunista de dieta ocasional omnívora, en la cual la proporción de los contenidos estomacales varía de acuerdo a los sitios de muestreo y el ciclo hidrobiológico.

\section{AGRADECIMIENTOS}

A la Gobernación del Vaupés por la financiación a través del Sistema General de Regalías (Convenio Especial de Cooperación 0032 de 2013), a la Universidad de los Llanos por la cofinanciación y a la Autoridad Nacional de Acuicultura y Pesca (AUNAP). Igualmente, a las comunidades indígenas de Santa Cruz, Yacayacá y Piracemo del departamento del Vaupés, Colombia. A Alfonso Velasco Santamaría, por su colaboración en la elaboración del mapa, a Víctor Atencio de la Universidad de Córdoba, por sus valiosos comentarios en la revisión del manuscrito.

\section{RESUMEN}

La especie más importante de la pesquería del río Vaupés es un pez conocido como Warakú tres puntos (Leporinus friderici), un carácido migratorio representativo del departamento del Vaupés, Colombia, de importancia para la seguridad alimentaria de comunidades indígenas. Con el propósito de contribuir en el conocimiento biológico básico de la especie, el presente estudio determinó los hábitos y preferencias alimenticias de Leporinus friderici durante un ciclo hidrobiológico (marzo 2014 a mayo 2015) en el río Vaupés, Colombia. Fueron muestreados tres lugares, dos a lo largo del río Vaupés (comunidades indígenas de Yacayacá y Santa Cruz) y uno en el río Cuduyarí afluente del río Vaupés (comunidad de Piracemo). Se analizaron 316 contenidos estomacales aplicando los métodos de frecuencia de ocurrencia (FO), índice gravimétrico (W), coeficiente de vacuidad (CV) e índice de importancia relativa (IIR). De igual modo, se determinaron parámetros físicos y químicos del agua superficial y profunda. La profundidad del río en época de aguas ascendentes fue de $3.7 \pm 0.6 \mathrm{~m}$, aguas altas de $5.9 \pm 1.4 \mathrm{~m}$, aguas descendentes $4.6 \pm 1.3 \mathrm{~m}$ y en aguas bajas $2.4 \pm 1.0 \mathrm{~m}$; se registró mayor acidez y turbidez del agua en las ascendentes. El CV fue de $14.5 \%$; sin embargo, el CV alcanzó un valor del $40 \%$ en aguas ascendentes. En general se observó una mayor FO de material vegetal en los estómagos de los individuos muestreados (44.4-66.7\%), seguido por los insectos (21.1-33.3\%, dietas secundarias). Los índices gravimétricos reflejaron que el material vegetal fue el ítem consumido en mayor cantidad, seguido de los insectos y en casi igual proporción invertebrados y material animal. Las diferencias en la composición de la dieta confirman la naturaleza oportunista de esta especie con predominio de hábitos omnívoros. 
Este estudio puede ser utilizado como parte integral del conocimiento de la ecología trófica de la especie, con el fin de crear estrategias para la protección del L. friderici en la región del Vaupés.

Palabras clave: amazonía, contenido estomacal, comunidades indígenas, hábitat, peces, Warakú.

\section{REFERENCIAS}

Agostinho, A. A., Hahn, N. S., Gomes, L. C., \& Bini, M. (1997). Estructura trófica. In A. E. A. de M. Vazzoler, A. A. Agostinho, \& N. S. Hahn (Eds.), A planície de inundação do alto rio Paraná. Aspectos físicos, biológicos e socioeconômicos (pp. 229-248). Maringá, Eduem.

Barón, C. (2006). Relaciones ecomorfológicas y de dieta en siete especies (Characidae) en afluentes de la quebrada Yahuarcaca (Amazonia colombiana) en un período de época seca (Tesis de grado). Universidad Javeriana, Bogotá. http://hdl.handle.net/10554/8871

Casas, J. Y., Lozano-Largacha, Y., \& Rivas, T. (2007). Contribución a la ecología trófica del dentón Leporinus muyscorum Steindachner 1902 en la ciénaga La Grande, cuenca media del río Atrato, Colombia. Revista Institucional Universidad Tecnológica del Chocó, 26, 4-8.

Castagnolli, N. (1992). Piscicultura de agua doce. Jaboticabal, FUNEP (p. 189).

Corporación Colombia Internacional \& Ministerio De Agricultura y Desarrollo Rural. (2009). Pesca y acuicultura Colombia 2009. Informe Técnico Regional Litoral Caribe y Pacífico. Bogotá. ISSN 2011-8139 (p. 1).

Durães, R., Pompeu, P. S., \& Godinho, A. L. (2001). Alimentação de quatro espécies de Leporinus (Characiformes, Anostomidae) durante a formação de um reservatório no sudeste do Brasil. Iheringia. Série Zoologia, 90, 183-191.

Franco, L. \& Bashirullah, K. M. B. (1992). Alimentación de la lisa (Mugil curema) del Golfo de Cariaco-Estado Suche, Venezuela. Zootecnia Tropical, 10(2), 219-238.

Garavello, J. C., \& Britski, H. A. (2003). Family Anostomidae. In REIS RE (Ed.), Checklist of the freshwater fishes of south and Central America (pp. 71-84). Porto Alegre: Editora da Pontifícia Universidade Católica do Rio Grande do Sul.

Hahn, N. S., Andrian, I. F., Fugi, R., \& De Almeida, V. L. (1997). Ecologia trófica. In A. E. A. de M. Vazzoler, A. A. Agostinho, \& N. S. Hahn (Eds.), A planície de inundação do alto rio Paraná. Aspectos fisicos, biológicos e socioeconômicos (pp. 209-228). Eduem: Maringá.
Hajisamaea, S., Choua, L. M., \& Ibrahimb, S. (2003). Feeding habits and trophic organization of the fish community in shallow waters of an impacted tropical hábitat. Estuarine, Coastal and Shelf Science, $58,89-98$.

Heinz, S., \& Dieter, K. (1987). Atlas de los microorganismos de agua dulce. Barcelona: Ediciones Omega. S.A.

Hyslop, E. J. (1980). Stomach content analysis, a review of methods and their application. Journal Fish Biology, 17(4), 411-430.

Kellnreitner, F., Pockberger, M., \& Asmus, H (2012). Seasonal variation of assemblage and feeding guild structure of fish species in a boreal tidal basin. Estuarine, Coastal and Shelf Science, 108, 97-108.

Krebs, C. J. (1989). Ecological Methodology. New York, USA: Harper and Row.

Lagler, K. F., Bardach, J. E., Miller, R. R., \& May-Passino, D. R. (1977). Ichthyology. 2da Edición. New York: John Wiley y Sons, Inc.

Lasso, C. (2004). Los peces de la Estación Biológica El Frío y Caño Guaritíco (Estado Apure), Llanos del Orinoco, Venezuela. Publicaciones del Comité Español del Programa Hombre y Biosfera-Red Ibero MAB, Unesco. Sevilla.

Lasso, C. A. \& Sánchez-Duarte, P. (2011). Los peces del delta del Orinoco. Diversidad, bioecología, uso y conservación. Caracas, Venezuela: Fundación La Salle de Ciencias Naturales y Chevron C. A.

Longart, Y., Acosta, V., Parra, B., \& Lista, M. (2011). Hábitos alimenticios del marao fósforo Hemirhamphus brasiliensis de los alrededores de la Isla de Cubagua, Venezuela. Zootecnia Tropical, 29(1), 77-87.

Maldonado-Ocampo, J. A., Ortega-Lara, A., UsmaOviedo, J. S., Galvis Vergara, G., Villa-Navarro, F. A., Vásquez-Gamboa, L., Prada-Pedreros, S., \& Ardila-Rodríguez, C. (2005). Peces de los Andes de Colombia. Instituto Humboldt, WWF-Colombia, Inciva, Universidad del Tolima, Universidad Javeriana, Universidad Nacional de Colombia, Universidad Metropolitana de Barranquilla, GEF, Banco Mundial, Embajada de los Países Bajos.

Mendonça, F. P., Magnusson, W. E., \& Zuanon, J. (2005). Relationships between habitat characteristics and fish assemblages in small streams of Central Amazonia. Copeia, 4, 751-764.

Needham, J. G. \& Needham, P. R. (1982). Guía para el estudio de los seres vivos de aguas dulces. Barcelona: Reverté. S.A.

Novoa, D. (2002). Los recursos pesqueros del eje fluvial Orinoco-Apure: presente y futuro. Ministerio de Agricultura y Tierras. Caracas: Insapesca. 
Pérez-Mayorga, M. A., \& Prada-Pedreros, S. (2011). Relación entre comunidad ictica y cobertura vegetal riparia en períodos hidrológicos (Eje Cafetero, Colombia). Universitas Scientiarum, Mayo-Agosto, 119-139.

PGAR. (2011). Plan de Gestión Ambiental de la "Región" del sur de la Amazonia Colombiana PGAR 20022011. Corporación para el desarrollo sostenible del Sur de la Amazonia (CORPOAMAZONIA).

Ramírez-Herrejón, J. P., Castañeda-Sam, L. S., MoncayoEstrada, R., Caraveo-Patiño, J., \& Balart, E. F. (2013). Trophic ecology of the exotic Lerma livebearer Poeciliopsis infans (Cyprinodontiformes: Poeciliidae) in the Lago de Pátzcuaro, Central México. Revista de Biología Tropical, 61(3), 1289-1300.

Roldan, P. G. \& Ramírez, R. J. J. (2008). Fundamentos de limnología neotropical. $2^{\text {a }}$ Edición. Medellín, Colombia: Editorial Universidad de Antioquia.

Santos, G. M. (1982). Caracterização, hábitos alimentares e reprodutivos de quatro espécies de "aracus" e considerações ecológicas sobre o grupo no lago Janauacá, AM (Osteichthyes, Characoidei, Anostomidae). Acta Amazonica, 12(4), 713-739.

Sostoa, A., Garcia De Jalón, D., \& Garcia-Berthou, E. (2005). Protocolos de muestreo y análisis para ictiofauna, p. 179-213. In De La Fuente, M. J. (Ed.), Metodología para el establecimiento del estado ecológico según la directiva marco del agua en la Confederación hidrográfica del Ebro. España: Ministerio del Medio Ambiente.

Steel, R. \& Torrie, J. (1988). Bioestadística: principios y procedimientos. $2^{\mathrm{a}}$ ed. México: McGraw-Hill.
Torres-Rojas, Y. E. (2011). Nicho trófico de pelágicos mayores capturados en la boca del Golfo de California (Tesis doctoral). México: Centro Interdisciplinario de Ciencias Marinas-Instituto Politécnico Nacional, La Paz Baja California Sur.

Tyler, J. E. (1968). The Secchi disc. Limnology and Oceanography, 13, 1-6.

Vazzoler, A. E. A. de M., Suzuki, H. I., Marques, E. E., \& Pérez-Lizama, M. de los A. (1997). Primeira maturação gonadal, períodos e áreas de reprodução. In A. E. A. de M. Vazzoler, A. A. Agostinho \& N. S. Hahn (Eds.), A planície de inundação do alto rio Paraná. Aspectos físicos, biológicos e socioeconómicos (pp. 249-266). Eduem: Maringá.

Villadiego, P., OrtizVillafañe, E., \& AtencioGarcía, V. J. (2004). Evaluación del régimen alimentario del bagre blanco Sorubim cuspicaudus (Pisces: Pimelodidae) en el bajo río Sinú, Colombia. Dahlia Revista Asociación Colombiana de Ictiología, 7, 1321.

Yáñez-Arancibia, A., Lara-Domínguez, A. L., AguirreLeón, A., Díaz-Ruiz, S., Amescua- Linares, F., Flórez-Hernández, D., \& Chavance, P. (1985). Ecología de poblaciones de peces dominantes en estuarios tropicales: Factores ambientales que regulan las estrategias biológicas y la producción. En A. YáñezArancibia (Ed.), Ecología de comunidades de peces en estuarios y lagunas costeras (pp. 311-365). México: D.F UNAM.

Zamudio, J., Urbano, A., Maldonado, J., Bogotá. J., \& Cortés, G. (2008). Hábitos alimentarios de diez especies de peces del piedemonte del departamento del Casanare, Colombia. Dahlia Revista Asociación Colombiana de Ictiología, 10, 43-55. 